\title{
An investigation on dynamic behavior of rotating shafts using a pipe elbow finite element formulation
}

\author{
M. Sajjadpour ${ }^{a}$ and S. A. Hosseini Kordkheilia ${ }^{*}$
}

${ }^{a}$ Aerospace Engineering Department, Sharif University of Technology, Azadi Avenue, P.O. Box: 11365-9567, Tehran, Iran

\begin{tabular}{l}
\hline A R T I C L E I N F O \\
\hline Article history: \\
Received 20 July 2021 \\
Accepted 11 December 2021 \\
Available online \\
16 January 2022 \\
\hline Keywords: \\
Dynamic behavior \\
Rotating shafts \\
Pipe elbow element \\
Finite Element Formulation \\
Critical speed \\
Natural frequency
\end{tabular}

\begin{abstract}
A B S T R A C T
Rotating shafts have a vast application in various industries especially in the aerospace industry such as engines, compressors and turbines. The researchers have performed considerable efforts on the rotating shafts' dynamic behavior because of their sensitivity to the rotor specifications and different parameters such as supports. In this paper by employing a pipe elbow element, an especial finite element formulation is derived to investigate dynamic behavior of rotating shaft in the presence of support clearance. The proposed element consists of four nodes with twenty-four degrees of freedom, which also accounts for the shear and gyroscopic effects. Within a finite element analysis framework, the focus of the paper is proposing a formulation to account for the dynamic behavior of a rotating shaft with much less number of elements. The element is implemented in a finite element code and then is used to model and analyze some rotating shaft examples. In order to verify the developed formulation, results are compared with those obtained from other schemes reported in the literature.
\end{abstract}

(C) 2022 Growing Science Ltd. All rights reserved.

\section{Introduction}

Due to the importance of using rotors or rotating shafts in various industries, the study of dynamic behavior of these shafts has always been an important part of engineering research. So far, many investigations have been performed for investigating the dynamic behavior of rotors using various analytical, semi-analytical and numerical methods. One of the numerical methods used to investigate rotors is the finite element method. Nelson and McVaugh (1976) presented a complete dynamic model of rotor-bearing systems including rigid discs, shafts discretized to finite elements and separate bearings based on the finite element method. They implemented the Euler-Bernoulli beam model to extract stiffness matrices. A few years later, Nelson (1980) used the Timoshenko beam model to develop finite element formulation of rotors, in which he could describe the effect of shear deformation and rotational inertia on the critical speeds. The Timoshenko beam model (Lien-Wen and Der-Ming, 1991) used a three-node beam element to analyze the critical speeds of the rotating shafts. (Nandi and Neogy, 2001), used three-dimensional solid finite elements to model and solve rotor dynamic problems, which automatically include shear deformations and rotational inertia due to the three-dimensional elasticity formulation. They modeled shafts and discs using the same type of element and this makes it possible to take into account the flexibility of the disc as well. (Jahromi et al., 2014), used a laboratory test rig to observe critical speed in a Jeffcott rotor. They also used the analytical method and the finite element method to validate the results. Sinou et al. (2005) investigated the effect of rotor bearing support flexibility on its dynamic behavior as well as its first critical forward and backward speeds. Tamrakar and Mittal (2016) obtained natural frequencies of un-cracked and cracked rotors in non-rotating conditions and critical speeds in the first and second modes in rotating conditions using both finite element and experimental schemes. They investigated the

* Corresponding author. Tel.:+98-21-6616-4609; fax: +98-21-6602-2731

E-mail addresses: ali.hosseini@sharif.edu (S. A. Hosseini Kordkheili) 
effects of crack properties on dynamic behavior of rotors by comparing Campbell diagrams. Torabi and Afshari (2016), derived an analytical solution for dynamic behavior and whirling analysis of axial-loaded Timoshenko rotors. They investigated the effect of angular velocity of spin, axial load, slenderness and Poisson's ratio on the natural frequencies of these rotors.

Tiwari (2017) used the Timoshenko beam element of a two-node with four degrees of freedom to calculate the first and second backward and forward critical speed of a continuous rotating shaft. Sousa et al. (2017), investigated the dynamic behavior of a flexible rotor by considering the basic excitation using both experimental and finite element methods. They considered the shaft as a Timoshenko beam and modeled it by a two-node beam element with eight degrees of freedom. Ishida et al. (2009) observed vibration characteristics in turbo machinery with radial clearance between a bearing outer ring and a casing. Their work primarily focused on the self-excited oscillation by numerical method. They modeled a flexible rotor to present where bearing collides with casing due to clearance, and derived its equation of motion. (Hosseini Kordkheili and Bahai, 2009) presented a finite element formulation for geometrically nonlinear analysis of structures. Their formulation was based on a shell element and an Updated Lagrangian (UL) formulation. They then extended this formulation and derived finite element formulation for a pipe elbow element to analyze riser structures (Hosseini Kordkheili et al., 2008).

This paper presents a finite element formulation based on a four-nodded, twenty-four degree of freedom annular section pipe elbow element to characterize the properties of the rotating shafts. This element can represent axial, shear, torsional and bending displacements as well as rotations. In order to show the accuracy and capability of the presented finite element formulation to model rotors, some examples are solved and the results are compared with those available in the literature.

\section{Kinematics of Pipe Elbow Element}

Fig. 1 depicts a four-nodded, twenty-four degrees of freedom three-dimensional annular section pipe elbow element that employed in the present paper. This element also considers shear deformations. According to Fig. 1, three coordinates $(r, s, t)$ are used to define the geometry of the element, where, the curvilinear coordinate $r$ expresses the element mid-line, and the two other coordinates express any position between the inner and the outer surfaces of the element section. Here each $k^{\text {th }}$ node also has three translations $\left(u_{1}^{k}, u_{2}^{k}, u_{3}^{k}\right)$ and three rotations $\left(\theta_{1}^{k}, \theta_{2}^{k}, \theta_{3}^{k}\right)$ DOFs with respect to the global $\left(x_{1}, x_{2}, x_{3}\right)$ coordinate system.

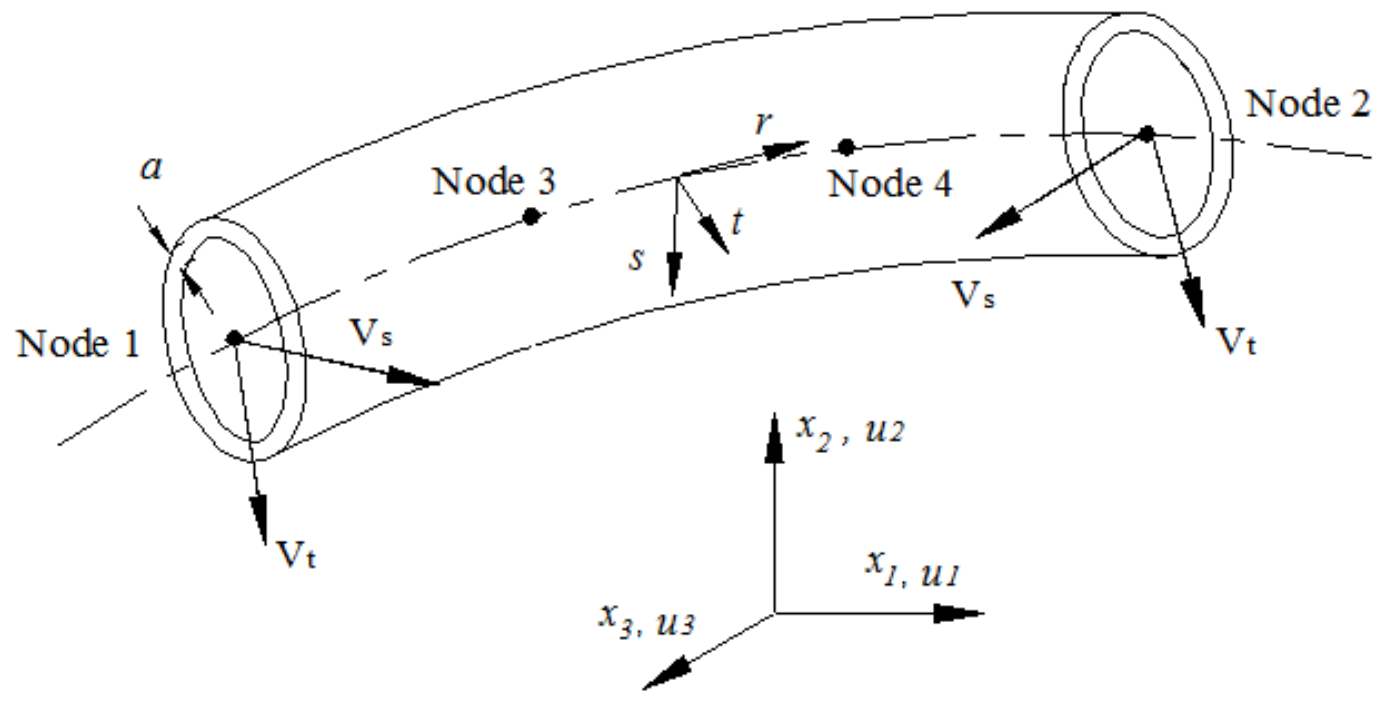

Fig. 1. Four-nodded pipe elbow element

After finite element discretization on the middle line of the continuum pipe elbow element, the configuration of this element having thickness $a^{k}$ for node $k$ is expressed as follows:

$$
x_{i}(r, s, t)=\sum_{k=1}^{4} h_{k}(r) x_{i}^{k}+s \sum_{k=1}^{4} h_{k}(r) a^{k} V_{s i}^{k}+t \sum_{k=1}^{4} h_{k}(r) a^{k} V_{t i}^{k} ; \quad i=1,2,3
$$

where $h_{k}(r)$ are cubic shape functions corresponding to nodal point $k$. Also, $V_{s i}^{k}$ and $V_{t i}^{k}$ are the local normal vector components at node $k$ in directions $s$ and $t$, respectively. Using Eq. (1), the displacement field of the element is obtained as: 
$u_{i}(r, s, t)=\sum_{k=1}^{4} h_{k}(r) u_{i}^{k}+t \sum_{k=1}^{4} a^{k} h_{k}(r) V_{t i}^{k}+s \sum_{k=1}^{4} a^{k} h_{k}(r) V_{s i}^{k} ; i=1,2,3$

Therefore

$$
\mathbf{U}=\mathbf{H}(r, s, t)\left\{\begin{array}{llllllll}
\cdots & u_{1}^{k} & u_{2}^{k} & u_{3}^{k} & \theta_{1}^{k} & \theta_{2}^{k} & \theta_{3}^{k} & \cdots
\end{array}\right\}^{T}=\mathbf{H}(r, s, t) \mathbf{u}^{k}
$$

where $\mathbf{U}$ is displacement vector and $\mathbf{H}(r, s, t)$ is displacement interpolation matrix which can be decomposed as below

$$
\mathbf{H}(r, s, t)=\mathbf{H}_{a}(r)+s \mathbf{H}_{b}(r)+t \mathbf{H}_{c}(r),
$$

Where

$$
\begin{aligned}
& \mathbf{H}_{a}(r)=\left[\cdots \mathbf{H}_{a}^{(k)}(r) \cdots\right]_{k=1 \ldots 4}, \mathbf{H}_{a}^{(k)}(r)=\left[\begin{array}{cccccc}
h_{k} & 0 & 0 & 0 & 0 & 0 \\
0 & h_{k} & 0 & 0 & 0 & 0 \\
0 & 0 & h_{k} & 0 & 0 & 0
\end{array}\right] \\
& \mathbf{H}_{b}(r)=\left[\cdots \mathbf{H}_{b}^{(k)}(r) \cdots\right]_{k=1 \ldots 4}, \mathbf{H}_{b}^{(k)}(r)=a_{k} h_{k}\left[\begin{array}{cccccc}
0 & 0 & 0 & 0 & V_{s 3}^{k} & -V_{s 2}^{k} \\
0 & 0 & 0 & -V_{s 3}^{k} & 0 & V_{s 1}^{k} \\
0 & 0 & 0 & V_{s 2}^{k} & -V_{s 1}^{k} & 0
\end{array}\right] \\
& \mathbf{H}_{c}(r)=\left[\cdots \mathbf{H}_{c}^{(k)}(r) \cdots\right]_{k=1 \ldots 4}, \mathbf{H}_{c}^{(k)}(r)=a_{k} h_{k}\left[\begin{array}{cccccc}
0 & 0 & 0 & 0 & V_{t 3}^{k} & -V_{t 2}^{k} \\
0 & 0 & 0 & -V_{t 3}^{k} & 0 & -V_{t 1}^{k} \\
0 & 0 & 0 & V_{t 2}^{k} & -V_{t 1}^{k} & 0
\end{array}\right]
\end{aligned}
$$

\section{Dynamic Finite Element Formulation for Rotating Shafts}

In order to derive dynamic finite element formulation for rotating shafts in presence of gyroscopic effects arising from the Coriolis acceleration, we consider the equations of Lagrange for the energies kinetic and potential of the solid that is given as follows

$$
\frac{d}{d t}\left(\frac{\partial \mathbf{K} . \mathbf{E} .}{\partial \dot{\mathbf{U}}}\right)-\frac{\partial \mathbf{K} . \mathbf{E} .}{\partial \mathbf{U}}+\frac{\partial \mathbf{P} . \mathbf{E} .}{\partial \mathbf{U}}=0
$$

where K.E. and P.E. are the kinetic and potential energies of the system, respectively, and can be written using the structural matrices as follows

$$
\begin{aligned}
\mathbf{K . E} . & =\frac{1}{2} \dot{\mathbf{U}}^{T} \mathbf{M} \dot{\mathbf{U}}+\frac{1}{2} \dot{\mathbf{U}}^{T} \mathbf{G} \dot{\mathbf{U}}-\frac{1}{2} \mathbf{U}^{T} \mathbf{N} \mathbf{U}-\mathbf{U}^{T} \int_{V} \rho \mathbf{H}_{(r, s, t)}^{T} \mathbf{\Omega}^{2} \mathbf{x} d V+\dot{\mathbf{U}}^{T} \int_{V} \rho \mathbf{H}_{(r, s, t)}^{T} \mathbf{\Omega} \mathbf{x} d V \\
& -\frac{1}{2} \int_{V} \rho \mathbf{U}^{T} \mathbf{\Omega}^{2} \mathbf{U} d V-\int_{V} \rho \mathbf{U}^{T} \boldsymbol{\Omega}^{2} \mathbf{U} d V+\int_{V} \rho \dot{\mathbf{U}}^{T} \mathbf{\Omega} \mathbf{x} d V-\frac{1}{2} \int_{V} \rho \mathbf{x}^{T} \mathbf{\Omega}^{2} \mathbf{x} d V \\
\text { P.E. }= & \frac{1}{2} \mathbf{U}^{T} \mathbf{K} \mathbf{U}-\mathbf{U}^{T} \int_{V} \mathbf{H}_{(r, s, t)}^{T} \mathbf{f} d V-\mathbf{U}^{T} \int_{V} \mathbf{H}_{(r, s, t)}^{T} \mathbf{t} d V
\end{aligned}
$$

where $\mathbf{M}$ is mass matrix, $\mathbf{K}$ is the linear stiffness matrix, $\mathbf{G}$ is Coriolis matrix, $\mathbf{N}$ is centrifugal acceleration matrix and $\boldsymbol{\Omega}$ is rotational matrix. Also, $\mathbf{x}$ is geometry vector, $\mathbf{f}$ denotes the body load vector and $\mathbf{t}$ is the surface traction vector. These matrices and vectors are obtained based on equilibrium equation as follows

$$
\mathbf{M}=\int_{V} \rho \mathbf{H}_{(r, s, t)}^{\mathrm{T}} \mathbf{H}_{(r, s, t)} d V
$$




$$
\begin{aligned}
& \mathbf{K}=\int_{V} \mathbf{B}^{\mathrm{T}}(r, s, t) \mathbf{C} \mathbf{B}(r, s, t) d V \\
& \mathbf{G}=2 \int_{V} \rho \mathbf{H}_{(r, s, t)}^{\mathbf{T}} \mathbf{\Omega} \mathbf{H}_{(r, s, t)} d V \\
& \mathbf{N}=\int_{V} \rho \mathbf{H}_{(r, s, t)}^{\mathbf{T}} \mathbf{\Omega}^{2} \mathbf{H}_{(r, s, t)} d V \\
& \mathbf{\Omega}=\left[\begin{array}{ccc}
0 & -\omega_{z} & \omega_{y} \\
\omega_{z} & 0 & -\omega_{x} \\
-\omega_{y} & \omega_{x} & 0
\end{array}\right]
\end{aligned}
$$

where $\mathbf{C}$ is the material properties matrix, $\omega_{x}, \omega_{y}$ and $\omega_{z}$ are rotating speeds about corresponding axes. $\mathbf{B}$ is straindisplacement matrix which can be decomposed as

$$
\mathbf{B}(r, s, t)=\mathbf{B}_{\mathbf{a}}(r)+s \mathbf{B}_{\mathbf{b}}(r)+t \mathbf{B}_{\mathbf{c}}(r)
$$

where $\mathbf{B}_{a}(r)=\left[\cdots \mathbf{B}_{a}^{(k)}(r) \cdots\right], \mathbf{B}_{b}(r)=\left[\cdots \mathbf{B}_{b}^{(k)}(r) \cdots\right]$ and $\mathbf{B}_{c}(r)=\left[\cdots \mathbf{B}_{c}^{(k)}(r) \cdots\right]$ for $k=1 \ldots 4$ and

$$
\begin{aligned}
\mathbf{B}_{a}^{(k)}(r) & =\left[\begin{array}{cccccc}
h_{k, 1} & 0 & 0 & \Phi_{111}^{k} & \Phi_{211}^{k} & \Phi_{311}^{k} \\
0 & h_{k, 2} & 0 & \Phi_{122}^{k} & \Phi_{222}^{k} & \Phi_{322}^{k} \\
0 & 0 & h_{k, 3} & \Phi_{133}^{k} & \Phi_{233}^{k} & \Phi_{333}^{k} \\
h_{k, 2} & h_{k, 1} & 0 & \Phi_{112}^{k}+\Phi_{121}^{k} & \Phi_{212}^{k}+\Phi_{221}^{k} & \Phi_{312}^{k}+\Phi_{321}^{k} \\
0 & h_{k, 3} & h_{k, 2} & \Phi_{123}^{k}+\Phi_{132}^{k} & \Phi_{223}^{k}+\Phi_{232}^{k} & \Phi_{323}^{k}+\Phi_{332}^{k} \\
h_{k, 3} & 0 & h_{k, 1} & \Phi_{113}^{k}+\Phi_{131}^{k} & \Phi_{213}^{k}+\Phi_{231}^{k} & \Phi_{313}^{k}+\Phi_{331}^{k}
\end{array}\right] \\
\mathbf{B}_{b}^{(k)}(r) & =\left[\begin{array}{llllll}
0 & 0 & 0 & \hat{\Phi}_{11}^{k} & \hat{\Phi}_{211}^{k} & \hat{\Phi}_{311}^{k} \\
0 & 0 & 0 & \hat{\Phi}_{122}^{k} & \hat{\Phi}_{222}^{k} & \hat{\Phi}_{322}^{k} \\
0 & 0 & 0 & \hat{\Phi}_{133}^{k} & \hat{\Phi}_{233}^{k} & \hat{\Phi}_{333}^{k} \\
0 & 0 & 0 & \hat{\Phi}_{112}^{k}+\hat{\Phi}_{121}^{k} & \hat{\Phi}_{212}^{k}+\hat{\Phi}_{221}^{k} & \hat{\Phi}_{312}^{k}+\hat{\Phi}_{321}^{k} \\
0 & 0 & 0 & \hat{\Phi}_{123}^{k}+\hat{\Phi}_{132}^{k} & \hat{\Phi}_{223}^{k}+\hat{\Phi}_{232}^{k} & \hat{\Phi}_{323}^{k}+\hat{\Phi}_{332}^{k} \\
0 & 0 & 0 & \hat{\Phi}_{113}^{k}+\hat{\Phi}_{131}^{k} & \hat{\Phi}_{213}^{k}+\hat{\Phi}_{231}^{k} & \hat{\Phi}_{313}^{k}+\hat{\Phi}_{331}^{k}
\end{array}\right] \\
\mathbf{B}_{c}^{(k)}(r) & =\left[\begin{array}{llllll}
0 & 0 & 0 & \bar{\Phi}_{111}^{k} & \bar{\Phi}_{211}^{k} & \bar{\Phi}_{311}^{k} \\
0 & 0 & 0 & \bar{\Phi}_{122}^{k} & \bar{\Phi}_{222}^{k} & \bar{\Phi}_{322}^{k} \\
0 & 0 & 0 & \bar{\Phi}_{133}^{k} & \bar{\Phi}_{233}^{k} & \bar{\Phi}_{333}^{k} \\
0 & 0 & 0 & \bar{\Phi}_{112}^{k}+\bar{\Phi}_{121}^{k} & \bar{\Phi}_{212}^{k}+\bar{\Phi}_{221}^{k} & \bar{\Phi}_{312}^{k}+\bar{\Phi}_{321}^{k} \\
0 & 0 & 0 & \bar{\Phi}_{123}^{k}+\bar{\Phi}_{132}^{k} & \bar{\Phi}_{223}^{k}+\bar{\Phi}_{232}^{k} & \bar{\Phi}_{323}^{k}+\bar{\Phi}_{332}^{k} \\
0 & 0 & 0 & \bar{\Phi}_{113}^{k}+\bar{\Phi}_{131}^{k} & \bar{\Phi}_{213}^{k}+\bar{\Phi}_{231}^{k} & \bar{\Phi}_{313}^{k}+\bar{\Phi}_{331}^{k}
\end{array}\right]
\end{aligned}
$$

Also $\Phi_{m i n}^{k}=\left(J_{n 2}^{-1} \hat{\phi}_{m i}^{k}+J_{n 3}^{-1} \bar{\phi}_{m i}^{k}\right) h_{k}, \hat{\Phi}_{\min }^{k}=J_{n 1}^{-1} \hat{\phi}_{m i}^{k} h_{k, r}$ and $\bar{\Phi}_{m i n}^{k}=J_{n 1}^{-1} \bar{\phi}_{m i}^{k} h_{k, r}$. Moreover, nonzero values for $\hat{\phi}$ and $\bar{\phi} \quad$ are $\quad \hat{\phi}_{12}^{k}=-\hat{\phi}_{21}^{k}=-0.5 a^{k} V_{s 3}^{k}, \quad \hat{\phi}_{13}^{k}=-\hat{\phi}_{31}^{k}=0.5 a^{k} V_{s 2}^{k}, \quad \hat{\phi}_{23}^{k}=-\hat{\phi}_{32}^{k}=-0.5 a^{k} V_{s 1}^{k}, \quad \bar{\phi}_{12}^{k}=-\bar{\phi}_{21}^{k}=-0.5 a^{k} V_{t 3}^{k}$, $\bar{\phi}_{13}^{k}=-\bar{\phi}_{31}^{k}=0.5 a^{k} V_{t 2}^{k}, \bar{\phi}_{23}^{k}=-\bar{\phi}_{32}^{k}=-0.5 a^{k} V_{t 1}^{k}$.

Using Eq. (9) and Eq. (10) into Eq. (8), a finite element formulation for dynamic analysis of rotating shafts is developed. By assuming constant rotating speed, this finite element formulation is 
$\mathbf{M} \ddot{\mathbf{U}}+\mathbf{G} \dot{\mathbf{U}}+(\mathbf{K}+\mathbf{N}) \mathbf{U}=\mathbf{F}$

Substitution of Eq. (4) and Eq. (16) into Eqs. (11-15) results in

$$
\begin{aligned}
& \mathbf{M}=\int_{V} \rho\left(\mathbf{M}_{a}(r)+s \mathbf{M}_{b}(r)+t \mathbf{M}_{c}(r)+s t \mathbf{M}_{d}(r)+s^{2} \mathbf{M}_{e}(r)+t^{2} \mathbf{M}_{f}(r)\right) d V \\
& \mathbf{K}=\int_{V}\left(\mathbf{K}_{a}(r)+s \mathbf{K}_{b}(r)+t \mathbf{K}_{c}(r)+s t \mathbf{K}_{d}(r)+s^{2} \mathbf{K}_{e}(r)+t^{2} \mathbf{K}_{f}(r)\right) d V \\
& \mathbf{G}=2 \int_{V} \rho\left(\mathbf{G}_{a}(r)+s \mathbf{G}_{b}(r)+t \mathbf{G}_{c}(r)+s t \mathbf{G}_{d}(r)+s^{2} \mathbf{G}_{e}(r)+t^{2} \mathbf{G}_{f}(r)\right) d V \\
& \mathbf{N}=2 \int_{V} \rho\left(\mathbf{N}_{a}(r)+s \mathbf{N}_{b}(r)+t \mathbf{N}_{c}(r)+s t \mathbf{N}_{d}(r)+s^{2} \mathbf{N}_{e}(r)+t^{2} \mathbf{N}_{f}(r)\right) d V
\end{aligned}
$$

where

$$
\begin{aligned}
& \mathbf{M}_{a}(r)=\mathbf{H}_{a}^{\mathrm{T}}(r) \mathbf{H}_{a}(r), \mathbf{K}_{a}(r)=\mathbf{B}_{a}^{\mathrm{T}}(r) \mathbf{C B}_{a}(r), \mathbf{G}_{a}(r)=\mathbf{H}_{a}^{\mathrm{T}}(r) \mathbf{\Omega} \mathbf{H}_{a}(r), \mathbf{N}_{a}(r)=\mathbf{H}_{a}^{\mathbf{T}}(r) \boldsymbol{\Omega}^{2} \mathbf{H}_{a}(r) \\
& \mathbf{M}_{b}(r)=\mathbf{H}_{a}^{\mathrm{T}}(r) \mathbf{H}_{b}(r)+\mathbf{H}_{b}^{\mathrm{T}}(r) \mathbf{H}_{a}(r), \mathbf{K}_{b}(r)=\mathbf{B}_{a}^{\mathrm{T}}(r) \mathbf{C B}_{b}(r)+\mathbf{B}_{b}^{\mathrm{T}}(r) \mathbf{C B}_{a}(r), \\
& \mathbf{G}_{c}(r)=\mathbf{H}_{a}^{\mathrm{T}}(r) \Omega \mathbf{H}_{c}(r)+\mathbf{H}_{c}^{\mathrm{T}}(r) \Omega \mathbf{H}_{a}(r), \mathbf{N}_{c}(r)=\mathbf{H}_{a}^{\mathrm{T}}(r) \Omega^{2} \mathbf{H}_{c}(r)+\mathbf{H}_{c}^{\mathrm{T}}(r) \Omega^{2} \mathbf{H}_{a}(r) \\
& \mathbf{M}_{d}(r)=\mathbf{H}_{b}^{\mathrm{T}}(r) \mathbf{H}_{c}(r)+\mathbf{H}_{c}^{\mathrm{T}}(r) \mathbf{H}_{b}(r), \mathbf{K}_{d}(r)=\mathbf{B}_{b}^{\mathrm{T}}(r) \mathbf{C} \mathbf{B}_{c}(r)+\mathbf{B}_{c}^{\mathrm{T}}(r) \mathbf{C} \mathbf{B}_{b}(r), \\
& \mathbf{G}_{d}(r)=\mathbf{H}_{b}^{\mathrm{T}}(r) \Omega \mathbf{H}_{c}(r)+\mathbf{H}_{c}^{\mathrm{T}}(r) \Omega \mathbf{H}_{b}(r), \mathbf{N}_{d}(r)=\mathbf{H}_{b}^{\mathrm{T}}(r) \Omega^{2} \mathbf{H}_{c}(r)+\mathbf{H}_{c}^{\mathrm{T}}(r) \Omega^{2} \mathbf{H}_{b}(r) \\
& \mathbf{M}_{e}(r)=\mathbf{H}_{b}^{\mathrm{T}}(r) \mathbf{H}_{b}(r), \mathbf{K}_{e}(r)=\mathbf{B}_{b}^{\mathrm{T}}(r) \mathbf{C B}_{b}(r), \mathbf{G}_{e}(r)=\mathbf{H}_{b}^{\mathrm{T}}(r) \Omega \mathbf{H}_{b}(r), \mathbf{N}_{e}(r)=\mathbf{H}_{b}^{\mathrm{T}}(r) \Omega^{2} \mathbf{H}_{b}(r) \\
& \mathbf{M}_{f}(r)=\mathbf{H}_{c}^{\mathrm{T}}(r) \mathbf{H}_{c}(r), \mathbf{K}_{f}(r)=\mathbf{B}_{c}^{\mathrm{T}}(r) \mathbf{C} \mathbf{B}_{c}(r), \mathbf{G}_{f}(r)=\mathbf{H}_{c}^{\mathrm{T}}(r) \Omega \mathbf{H}_{c}(r), \mathbf{N}_{f}(r)=\mathbf{H}_{c}^{\mathrm{T}}(r) \Omega^{2} \mathbf{H}_{c}(r)
\end{aligned}
$$

Employing a certain order of Gaussian quadrature numerical integration method on element volume, Eqs. (21-24) are estimated. In order to make the formulation more efficient and to avoid higher order numerical integrations, and to eliminate some terms in Eqs. (21-24), an analytical explicit integration scheme for through-the-thickness coordinates $r$ and $t$ is employed to solve these equations. Thus, we conclude:

$$
\begin{aligned}
& \mathbf{M}=\int_{-1}^{1} \rho\left(a_{0} \mathbf{M}_{a}(r)+a_{1}\left[\mathbf{M}_{e}(r)+\mathbf{M}_{f}(r)\right]\right)\left|\mathbf{J}_{(r), s=0, t=0}\right| d r \\
& \mathbf{K}=\int_{-1}^{1}\left(a_{0} \mathbf{K}_{a}(r)+a_{1}\left[\mathbf{K}_{e}(r)+\mathbf{K}_{f}(r)\right]\right)\left|\mathbf{J}_{(r), s=0, t=0}\right| d r \\
& \mathbf{G}=2 \int_{-1}^{1} \rho\left(a_{0} \mathbf{G}_{a}(r)+a_{1}\left[\mathbf{G}_{e}(r)+\mathbf{G}_{f}(r)\right]\right)\left|\mathbf{J}_{(r), s=0, t=0}\right| d r \\
& \mathbf{N}=2 \int_{-1}^{1} \rho\left(a_{0} \mathbf{N}_{a}(r)+a_{1}\left[\mathbf{N}_{e}(r)+\mathbf{N}_{f}(r)\right]\right)\left|\mathbf{J}_{(r), s=0, t=0}\right| d r
\end{aligned}
$$

where

$$
a_{0}=\pi\left(1-\left(r_{i} / r_{o}\right)^{2}\right), a_{1}=\frac{\pi}{4}\left(1-\left(r_{i} / r_{o}\right)^{4}\right), a_{2}=\frac{\pi}{24}\left(1-\left(r_{i} / r_{o}\right)^{6}\right)
$$

To evaluate these matrices a three points Newton-Cotes scheme numerical integration is also adopted employed during finite element solution.

\section{Results and Discussion}

The dynamic finite element formulation of the rotating shafts derived in this paper has been implemented in an own developed code. Using this tool, some sample problems are solved and performance of the present finite element formulation is assessed.

\section{Modal Analysis of a Free-Free Hollow Conical Beam}

In the first example, a modal analysis is conducted on the hollow aluminum beam of Fig. 2a with a conical section using ten pipe elbow elements (Fig. 2b). Support condition is free - free and geometrical information of this beam is given in references (Nandi \& Neogy, 2001; Vest \& Darlow, 1989; Stephenson \& Rouch, 1993). Vest and Darlow (1989) measured 
natural frequencies of this beam both experimentally and numerically using a modulus-corrected beam element. Stephenson and Rouch (1993) also proposed an axisymmetric solid finite element formulation to calculate natural frequencies of this beam.

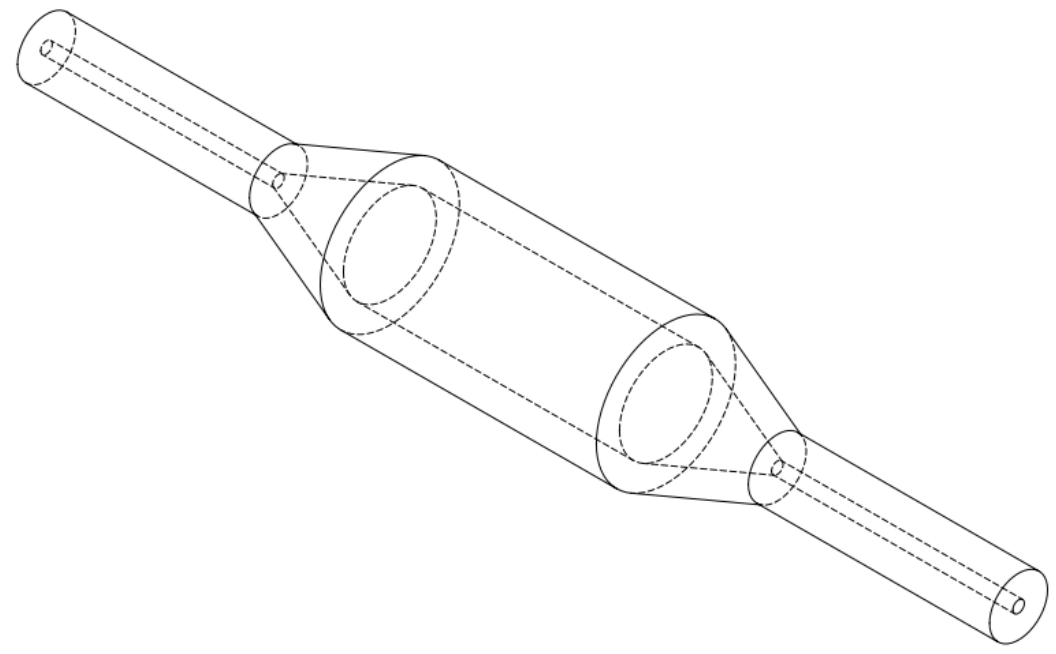

(a)

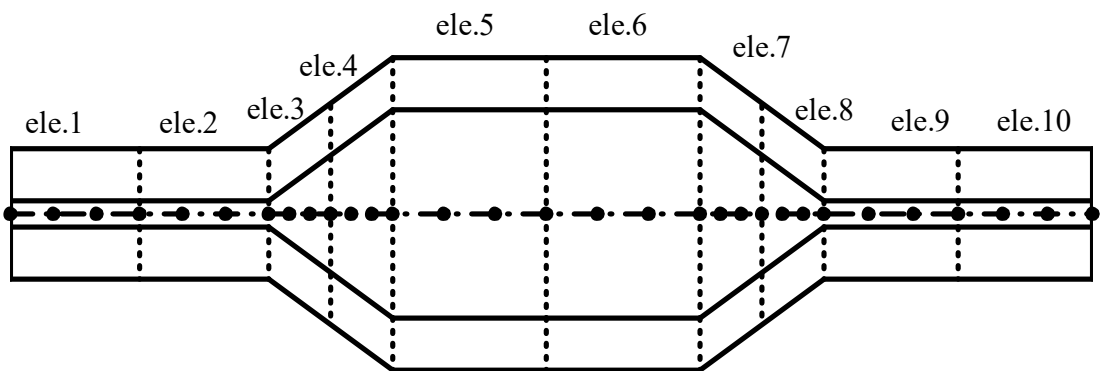

(b)

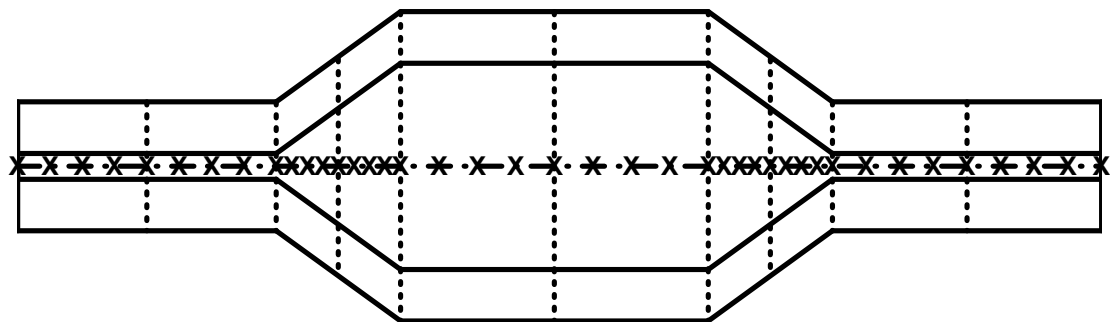

(c)

Fig. 2. a) A hollow aluminum beam with conical section, b) discretization using 10 pipe elbow elements together with 31 nodes, c) Five integration points

Table 1. Natural frequencies of hollow aluminum beam

\begin{tabular}{|c|c|c|c|c|c|c|}
\hline \multirow[b]{2}{*}{ Mode } & \multicolumn{3}{|c|}{ Present Work } & \multirow{2}{*}{$\begin{array}{c}\text { Beam element } \\
\text { (Vest and Darlow } \\
1989)\end{array}$} & \multirow{2}{*}{$\begin{array}{c}\text { BD } \\
625 \text { elements } \\
\text { (Nandi, \& Neogy 2001) }\end{array}$} & \multirow{2}{*}{$\begin{array}{c}\text { Experimental } \\
\text { (Vest and } \\
\text { Darlow 1989) } \\
\end{array}$} \\
\hline & 5 elements & 10 & 15 & & & \\
\hline 1 & 1045.7 & 1042.1 & 1042.0 & 1043.8 & 1052.9 & 1040 \\
\hline 2 & 1649.3 & 1644.8 & 1644.4 & 1658.7 & 1639.4 & 1643 \\
\hline 3 & 4144.8 & 4057.7 & 4054.8 & 4123.0 & 4056.7 & 4042 \\
\hline 4 & 6275.9 & 5829.4 & 5823.0 & 5817.0 & 5911.8 & 5886 \\
\hline 5 & 8529.3 & 7648.7 & 7645.3 & 7867.0 & 7590.2 & 7459 \\
\hline
\end{tabular}

Table 1 lists and compares the first five natural frequencies of the beam, those from the present finite element formulation and others reported in literature. In this table, one can observe that, with much less number of elements and employing a five integration point scheme (Fig. 2c), the results from the present work are more comparable with those from experiments. For example, in comparison with (Nandi and Neogy, 2001) work, we used more than 40 times lesser elements to solve this problem which greatly reduces computational costs. Moreover, it also observes that similar to other results, for higher mode 
numbers, the difference between the natural frequency values obtained from experiments and numerical solution slightly increases. However, the maximum divergence from experiment is about $2.6 \%$.

\section{Natural frequencies and critical speeds of a simply supported shaft}

For this example, natural frequencies and the forward and backward critical speeds of a uniform cross section simply supported spinning mild steel shaft are obtained using the developed pipe elbow finite element formulation.

The values of the natural frequencies of the first and second modes for the shaft with slenderness ratio of $\mathrm{R}=0.02(\mathrm{R}=\mathrm{r} / 2 \mathrm{~L}$, where $r$ is radius and $\mathrm{L}$ is length of the beam) are examined with different number of elements and are given and compared with (Shames and Dym, 2006) work in Table 2. According to these tabulated data, employing 10 elements leads to an acceptable accuracy for the results, so this discretization is used for further study. Non-dimensional critical speeds of the shaft (i.e. $\left.\bar{\omega}_{n}=\left[\rho l^{2} \omega_{n}{ }^{2} / E(\mathrm{R} / 2 l)^{2}\right]^{1 / 4}\right)$ with slenderness parameters 0.02 to 0.10 are then calculated and gathered in Table 3. In this table, the results are compared with those published by Eshleman and Eubanks (1969). From these results, it is noted that, the results from the present work are comparable with others in the literature with maximum $2.5 \%$ difference.

Table 2. Dimensionless non-rotating first and second mode natural frequencies of simply supported shaft

\begin{tabular}{ccccccc}
\hline & \multicolumn{5}{c}{ Present work } & \\
\cline { 2 - 5 } Mode & 1 Element & 2 Elements & 4 Elements & 10 Elements & 20 Elements & Closed form solution (Shames and Dym, 2006) \\
\hline 1 & 3.29018 & 3.13735 & 3.13260 & 3.13238 & 3.13238 & 3.1325 \\
2 & 6.52343 & 6.47782 & 6.21800 & 6.21165 & 6.21160 & 6.2122 \\
\hline
\end{tabular}

Table 3. Dimensionless non-rotating, first forward and backward critical speeds of simply supported shaft for different slenderness ratio

\begin{tabular}{cccccc}
\hline & Non rotating & \multicolumn{2}{c}{ 1st backward Critical Speed } & 1st forward Critical Speed \\
\hline \multirow{2}{*}{$\mathrm{R}$} & Present work & $\begin{array}{c}\text { Analytical } \\
\text { (Shames \& Dym, 2006) }\end{array}$ & Present work & $\begin{array}{c}\text { Analytical } \\
\text { (Eshleman \& Eubanks, 1969) }\end{array}$ & $\begin{array}{c}\text { Present work } \\
(\text { Eshleman \& Eubanks, 1969) }\end{array}$ \\
\hline 0.02 & 3.1324 & 3.1325 & 3.0547 & 3.1251 & 3.2184 \\
0.04 & 3.1058 & 3.1061 & 3.0286 & 3.0780 & 3.1891 \\
0.06 & 3.0646 & 3.0644 & 2.9896 & 3.0067 & 3.1455 \\
0.08 & 3.0125 & 3.0103 & 2.9400 & 2.9193 & 3.1245 \\
0.1 & 2.9531 & 2.9472 & 2.8833 & 2.8234 & 3.1037 \\
\hline
\end{tabular}

\section{Analyzing a cantilever beam with a disk in its free end}

In this example, a massless cantilever beam with a $1.815 \mathrm{~kg}$ disk in its free end is analyzed. The dimensions of this steel shaft and disk have been given in (Nandi \& Neogy, 2001; Thomson, 1981). According to Fig. 3, four elements (three elements for the shaft and one element for the disk) are employed to discretize the problem. The first non-rotating natural frequency of this system is calculated using a number of different elements. The disk is considered as one pipe element, the shaft is also divided into 2, 4 and 8 elements, and their results are given in Table 4. Furthermore, after selecting the appropriate number of elements, i.e. 4 elements, the Campbell diagram for this problem is depicted in Fig. 4 and compared with the analytical results reported in (Thomson, 1981). From this figure, one can see that the obtained results as well as the results from the closed form solution are in good agreement.

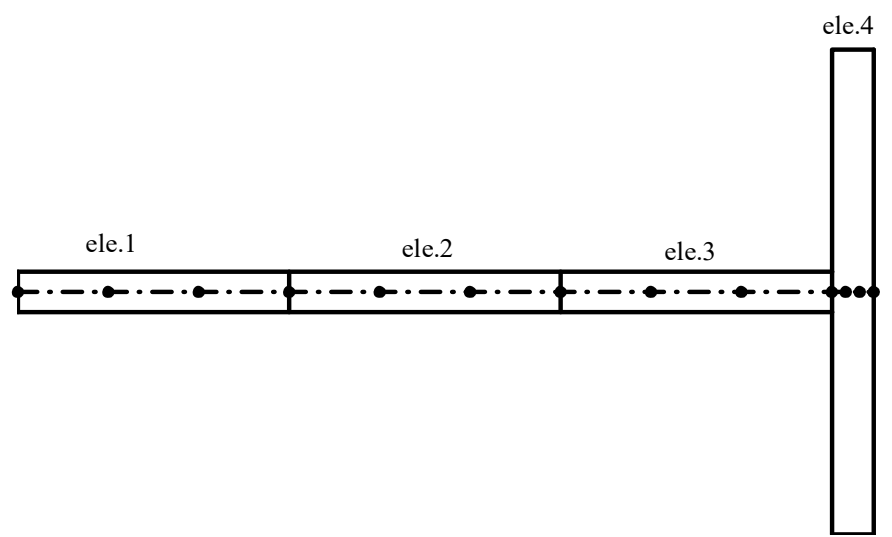

Fig. 3. A cantilever beam with a disk at its end (elements and nodes) 
Table 4. Natural frequencies of non-rotating cantilever beam with a disk in its free end ( $\mathrm{rad} / \mathrm{s})$

\begin{tabular}{cccccccc} 
& & \multicolumn{9}{c}{} & \multicolumn{2}{c}{ 3D FEM } \\
& Mode & 2 Elements & 3 Elements & 4 Elements & 11 Elements & 20 Elements & Analytical \\
(Thomson, 1981)
\end{tabular}

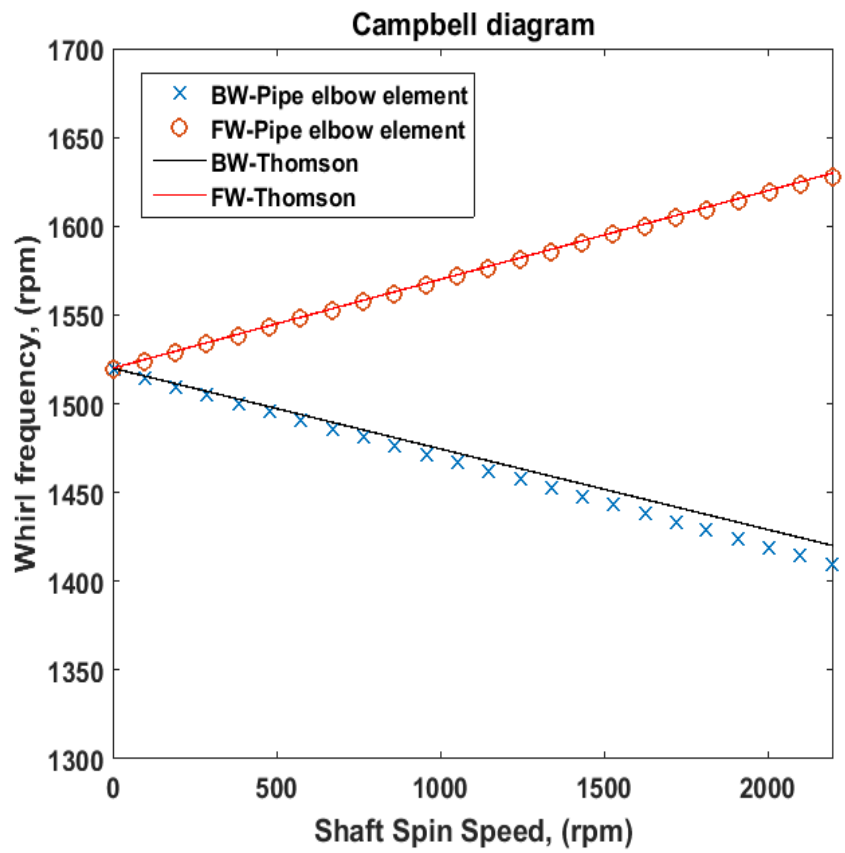

Fig. 4. Campbell diagram of the cantilever beam with a disk in its free end

\section{A simply supported shaft with a disk in the middle}

In this example, a simply supported steel shaft with length $400 \mathrm{~mm}$ and diameter $6 \mathrm{~mm}$ is analyzed. A disk with diameter $80 \mathrm{~mm}$ and thickness $25 \mathrm{~mm}$ is also located in the middle of the shaft (Fig. 5). The disk is modeled using one element and the shaft is divided into 2, 4 and 8 elements. In Table 5 the critical forward and backward speeds for the first mode are calculated with the number of different elements and compared with the experimental results obtained by (Jahromi et al., 2014). Results reveal that, due to inherent limitations in the measuring equipment, however, experiments executed by (Jahromi et al., 2014) could not separate forward and backward critical speeds of the shaft but our formulation leads to two different values for these speeds, which are in accordance with the experimental value.

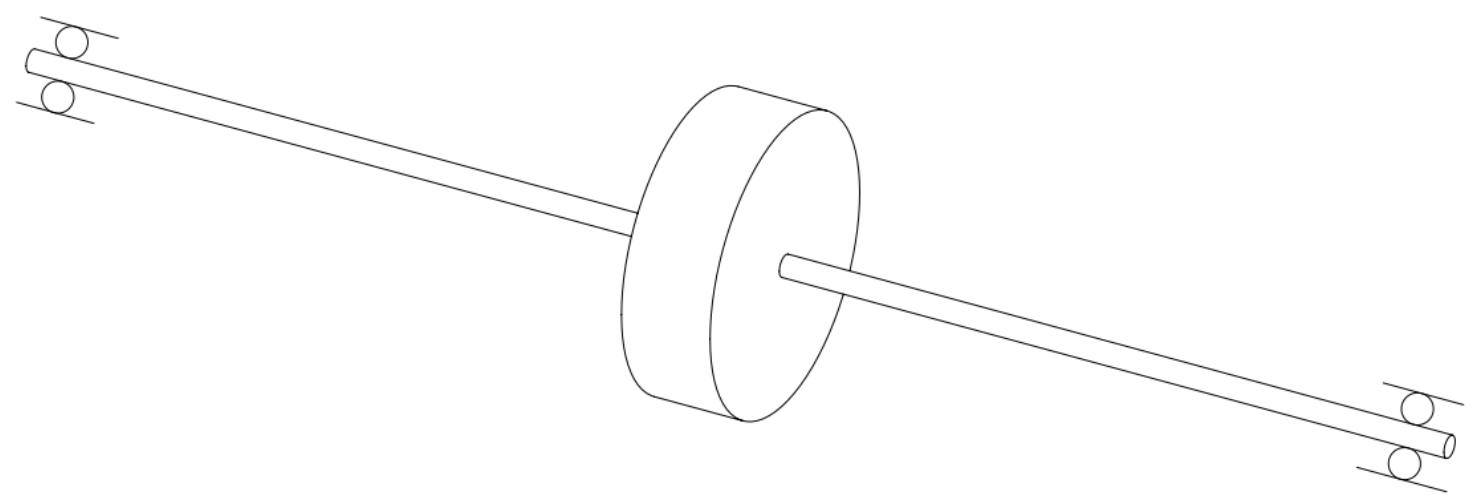

Fig. 5. A simply supported shaft with a disk in the middle 
Table 5. First critical forward and backward speeds of a simply supported shaft with a disk in the middle ( $\mathrm{rad} / \mathrm{s})$

\begin{tabular}{|c|c|c|c|c|}
\hline \multirow[b]{2}{*}{ Mode } & \multicolumn{3}{|c|}{ Present Work } & \multirow{2}{*}{$\begin{array}{c}\text { Experimental } \\
\text { (Jahromi et al., } \\
\text { 2014) } \\
\end{array}$} \\
\hline & 3 Elements & 5 Elements & 9 Elements & \\
\hline $\begin{array}{l}\text { 1st backward } \\
\text { Critical Speed }\end{array}$ & 107.6300 & 107.6221 & 107.4476 & 120.511 \\
\hline $\begin{array}{c}\text { 1st forward Critical } \\
\text { Speed }\end{array}$ & 119.5237 & 119.5159 & 119.3211 & 120.511 \\
\hline
\end{tabular}

\section{The bearing clearance effects of dynamic behavior of rotor}

Consider a vertical steel shaft with length $900 \mathrm{~mm}$ and diameter $16 \mathrm{~mm}$ in which a disc with a diameter $480 \mathrm{~mm}$ and thickness $5.5 \mathrm{~mm}$ is mounted on $350 \mathrm{~mm}$ distance from one of its supports (Fig. 6). The supports are ball bearings and the ball bearing of one of the supports has a radial clearance of $0.1 \mathrm{~mm}$ relative to the casing in which it is installed. (Ishida et al., 2009) investigated this problem by experimental and analytical methods. In this paper, this problem is solved by using a pipe elbow element and then the results are compared with their results for validation.

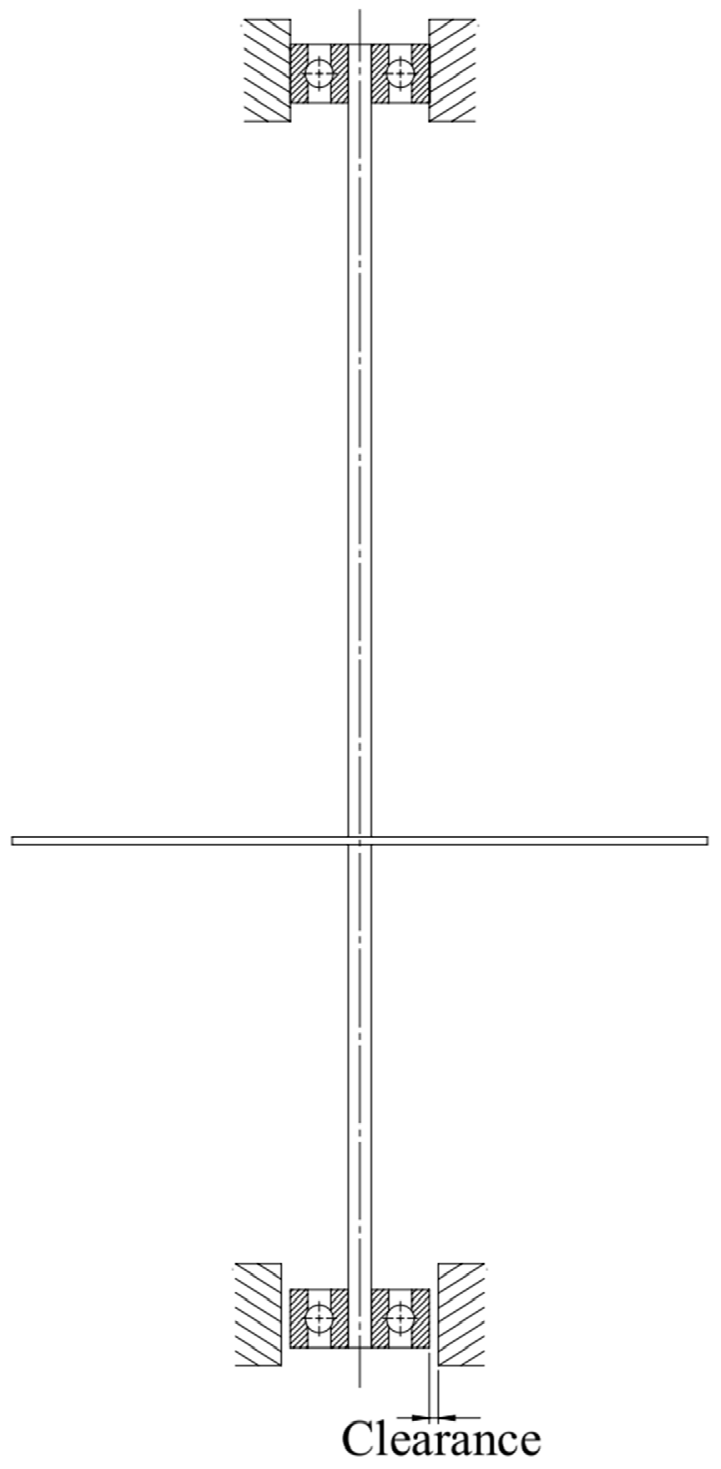

Fig. 6. A vertical rotor-bearing shaft with radial clearance 
Assuming the shaft to be simply supported, Campbell diagram is extracted and depicted in Fig. 7. From this diagram, the major critical speed is about $700 \mathrm{rpm}$ which is the same as reported by (Ishida et al., 2009).

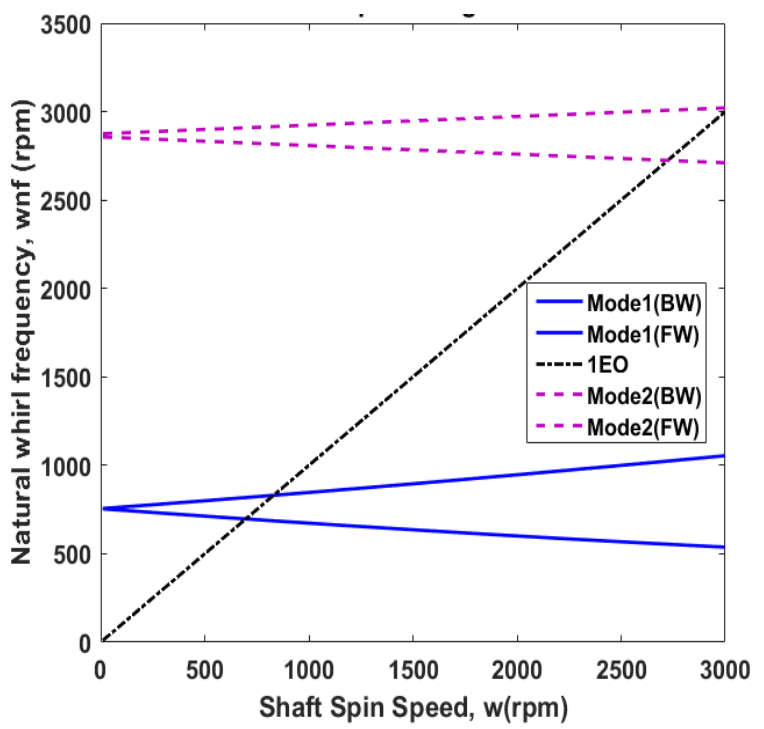

Fig. 7. Campbell diagram for the vertical shaft with simply supported boundary conditions

Now in order to consider and model the clearance in the lower bearing, the boundary condition at the lower end is considered to be free, i. e. the shaft is released at all degrees of freedom at this end. However, the movement of this end is limited according to the amount of clearance, i. e. $0.1 \mathrm{~mm}$. The collision between the bearing and the casing is shown in Fig. 8 .

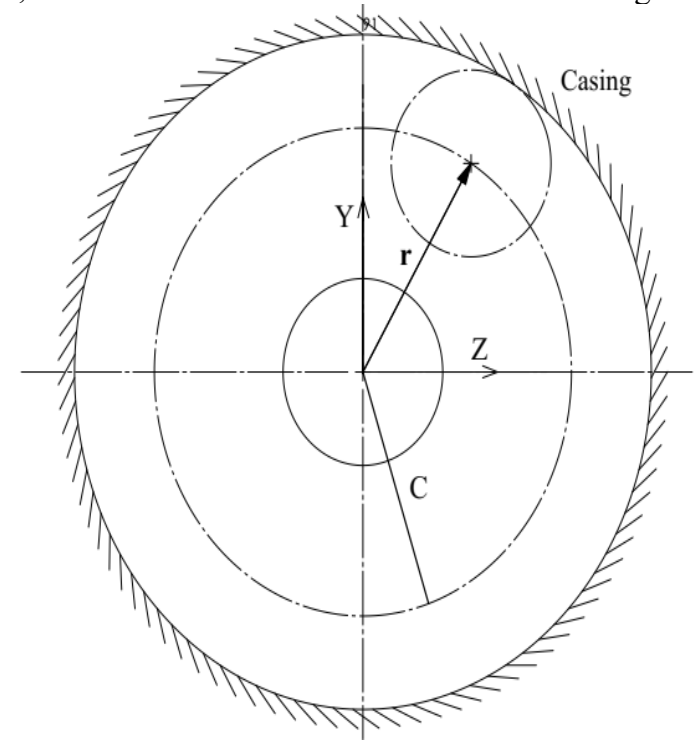

Fig. 8. Collision between bearing and casing due to clearance

In this figure, the bearing at its initial and current positions are shown much smaller than the casing to give more clarity. Taking radial movement of the bearing to be $r$ and clearance value to be $C$, simply we can say that if $r$ value comes to be greater than $C$ then the collision happens and these conditions are occurred; $v_{n+}=-e v_{n-}$ and $v_{t+}=v_{t_{-}}$; where $v_{n}$ and $v_{t}$ are the components of velocity in the vertical and tangential directions, respectively. The subscripts " - " and "+ " indicate the velocity values before and after collision, respectively. $e$ is the coefficient of restitution, which is determined experimentally.

Ishida et al. (2009) used the harmonic balance method, obtained orbit diagrams and displacement time history for the disk at four different speeds $1200 \mathrm{rpm}, 1321 \mathrm{rpm}, 1970 \mathrm{rpm}$ and $2671 \mathrm{rpm}$, and verified the results by tests. They considered both shaft and bearing damping properties and ignored their mass. They used two degrees of freedom for the disk and two degrees of freedom for the bearings to solve the problem. 
Here, adapting the developed pipe element formulation, we solve this problem to achieve the shaft behavior at the same four different speeds (Fig. 9). Comparing the results in this work with those reported by (Ishida et al., 2009) one can observe that the results coincide. Some visible differences may occur due to different initial conditions and assumptions. For example, in this work no damping is considered for either the shaft or the bearing as well as the shaft is continuously analyzed according to its mass. In the disk orbit diagram, from Fig. 9 one can see initial displacements as well as the displacement during transition state as well. However, (Ishida et al., 2009) did not report these displacements in their work.

(a)

$\omega=$ 1200rpm

(b)

$\omega=$

$1321 \mathrm{rpm}$

(c)

$\omega=$

1970rpm

(d)

$\omega=$

2671rpm
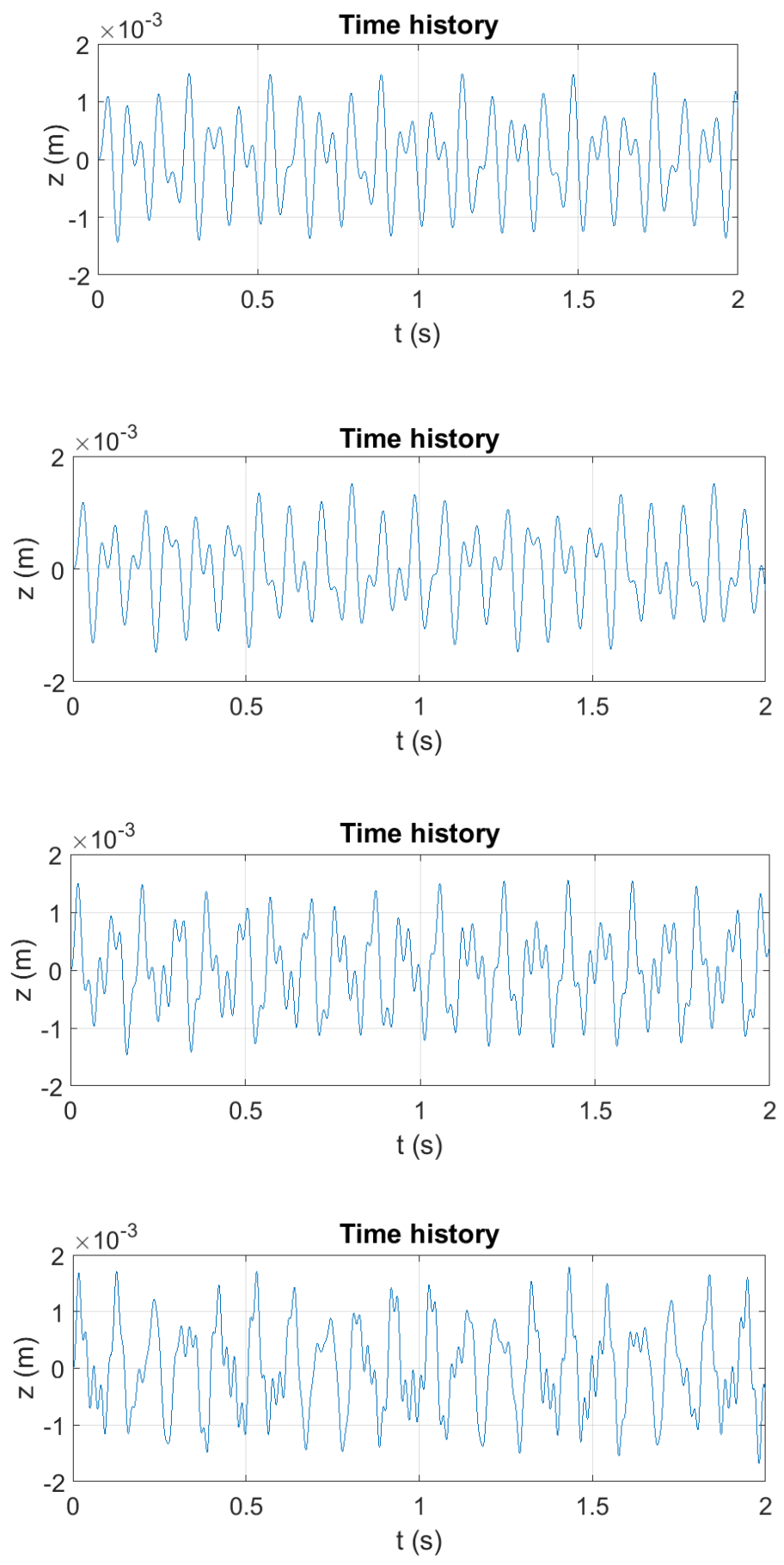
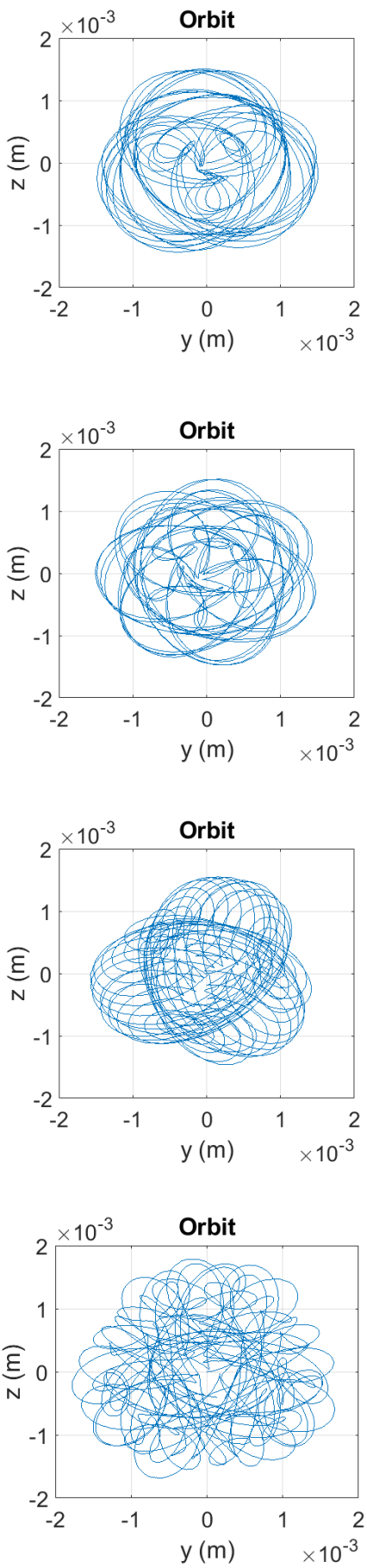

Fig. 9. Disk displacement time history and orbit

\section{Conclusions}

In this paper, a finite element formulation was presented for dynamic analysis of rotating shaft structures in presence of Coriolis effects. Based on the equations of Lagrange for the kinetic and potential energies of the rotating shaft, the dynamic 
formulation has been derived for an annular section three-dimensional pipe elbow element. Decomposing the displacement interpolation matrix in axial and transverse directions, made it possible to explicitly account governing equation matrices in the direction along the thickness. Moreover, these decompositions led to a particular formulation. It is noted that the employed integration scheme avoids inaccuracies normally associated with numerical integration methods during approximation of these matrices.

Some examples were solved and the results were compared with those available in the literature. It was shown that the results make good agreement with others in the literature after much reduced computational efforts. According to the results, the present more accurate formulation is capable to being adapted to solve rotating shafts with clearance in their supports.

\section{References}

Eshleman, R. L., \& Eubanks, R. A. (1969). On the critical speeds of a continuous rotor. Journal of Engineering Industry, 91(4), 1180-1188

Hosseini Kordkheili, S. A., \& Bahai, H. (2008, January). Nonlinear Dynamic Analysis of Flexible Riser Structures. In International Conference on Offshore Mechanics and Arctic Engineering (Vol. 48203, pp. 693-698).

Ishida, Y., Inagaki, M., Ejima, R., \& Hayashi, A. (2009). Nonlinear resonances and self-excited oscillations of a rotor caused by radial clearance and collision. Nonlinear Dynamics, 57(4), 593-605.

Jahromi, A. F., Bhat, R. B., \& Xie, W. F. (2015). Forward and backward whirling of a rotor with gyroscopic effect. In Vibration Engineering and Technology of Machinery (pp. 879-887). Springer, Cham.

Kordkheili, S. H., Naghdabadi, R., \& Jabbarzadeh, M. (2008). A geometrically nonlinear finite element formulation for shells using a particular linearization method. Finite elements in analysis and design, 44(3), 123-130.

Lien-Wen, C., \& Der-Ming, K. (1991). Finite element analysis of natural whirl speeds of rotating shafts. Computers \& Structures, 40(3), 741-747.

Nandi, A., \& Neogy, S. (2001). Modelling of rotors with three-dimensional solid finite elements. The Journal of Strain Analysis for Engineering Design, 36(4), 359-371.

Nelson, H. D. (1980). A finite rotating shaft element using Timoshenko beam theory. Journal of Mechanical Design, 102(4), 793-803

Nelson, H. D., \& McVaugh, J. M. (1976). The dynamics of rotor-bearing systems using finite elements. Journal of Engineering Industrial, 98(2), 593-600.

Shames, I. H., \& Dym, C. L. (2017). Energy and finite element methods in structural mechanics: SI units edition. Routledge.

Sinou, J. J., Villa, C., \& Thouverez, F. (2005). Experimental and numerical investigations of a flexible rotor on flexible bearing supports. International Journal of Rotating Machinery, 3, 179-189.

Sousa, M. S., Del Claro, V. T. S., Cavalini, A. A., \& Steffen, V. (2017). Numerical investigation on the dynamic behavior of an onboard rotor system by using the fem approach. Journal of the Brazilian Society of Mechanical Sciences and Engineering, 39(7), 2447-2458.

Stephenson, R. W., \& Rouch, K. E. (1993). Modeling rotating shafts using axisymmetric solid finite elements with matrix reduction.

Tamrakar, R., \& Mittal, N. (2016). Campbell diagram analysis of open cracked rotor. Engineering Solid Mechanics, 4(3), 159-166.

Thomson, W. T. (1981). Theory of Vibrations with Applications, Prentice-Hall Inc., Englewood Cliffs, NJ 07632.

Tiwari, R. (2017). Rotor systems: analysis and identification. CRC press.

Torabi, K., \& Afshari, H. (2016). Exact solution for whirling analysis of axial-loaded Timoshenko rotor using basic functions. Engineering Solid Mechanics, 4(2), 97-108.

Vest, T. A., \& Darlow, M. S. (1990). A modified conical beam element based on finite element analysis: experimental correlations. Journal of Vibration Acoustic, 112(3), 350-354

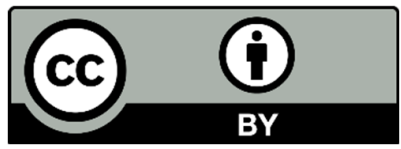

(C) 2022 by the authors; licensee Growing Science, Canada. This is an open access article distributed under the terms and conditions of the Creative Commons Attribution (CC-BY) license (http://creativecommons.org/licenses/by/4.0/). 